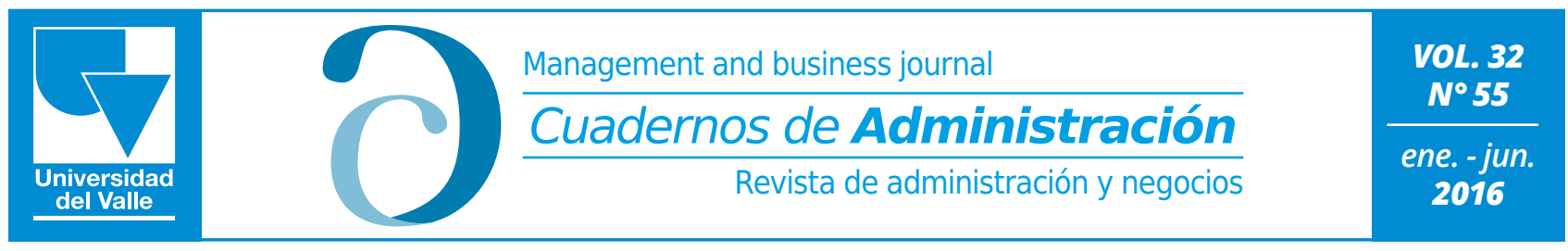

Print ISSN: 0120-4645 / E-ISSN: 2256-5078 / Short name: cuad.adm. / Pages: 19-32

Universidad del Valle / Facultad de Ciencias de la Administración / Universidad del Valle / Cali - Colombia

\title{
Análisis econométrico de la rentabilidad de los inversionistas en la industria panificadora y de pastas colombiana, 2000-2013
}

\author{
Econometric analysis of the profitability of investors in the bakery and pasta Colombian \\ industry, 2000-2013
}

\section{Analyse économétrique de la rentabilité des investisseurs dans l'industrie de la boulangerie et des pâtes, Colombie 2000-2013}

Luis D. Delgado Véleza

Docente investigador, Departamento de Contaduría, Corporación Universitaria Adventista de Colombia, Medellín, Colombia. E-mail: luis.delgado@esumer.edu.co

Research article, PUBLINDEX-COLCIENCIAS clasification
Submmit: 01/04/2016
Review: $18 / 04 / 2016$
Accepted: $15 / 06 / 2016$
Eje temático: finanzas

\section{Resumen}

La industria panificadora y de pastas en Colombia representa una de las actividades más importantes del sector manufactura, con unas ventas cercanas a 2,5 billones de pesos anuales. No obstante, algunos factores críticos como los precios de las materias primas en los mercados internacionales, aspectos culturales, la llegada de fuertes competidores transnacionales, y las adversidades propias de la industria nacional potencializan los riesgos para sus inversionistas. La rentabilidad del patrimonio (ROE) evidencia una reducción progresiva al pasar de 14,1\% en 2000 a 4,4\% en 2013; asimismo, el modelo de regresión lineal confirma este comportamiento, explicado en gran medida por los cambios en el margen bruto y la rentabilidad de sus activos. De otro lado, un aumento en los precios de venta ajustados a la inflación y una reducción sustancial en el consumo per cápita de pan en los 13 últimos años, al pasar de 32 a 24 kilos-habitante-año, indican un pobre desempeño de los ingresos y el uso de sus activos fijos. No obstante, el comportamiento de los costos de materias primas y mano de obra permite inferir que los empresarios controlaron los costos de producción, a pesar de la volatilidad en los precios internacionales del trigo.

Palabras clave: competitividad, diagnóstico financiero, econometría financiera, industria panificadora y de pastas, rentabilidad.

\footnotetext{
Abstract

The bakery and pasta industry in Colombia represents one of the most important activities in the manufacturing sector, with sales of nearly 2.5 trillion pesos, annually. However, some critical factors such as the prices of raw materials in international markets, cultural aspects, the arrival of strong multinational competitors and the adversities own of the domestic industry potentiate risks to their investors. The return on equity (ROE) shows a progressive reduction going from $14.1 \%$ in 2000 to $4.4 \%$ in 2013; in addition, the linear regression model confirms this behavior, largely explained

a Ingeniero Industrial, Universidad de Antioquia, Medellín Colombia. Magister en Finanzas, Universidad Pablo de Olavide, Sevilla, España. Grupo de Investigación en Estudios Organizacionales, clasificación D Colciencias, Corporación Universitaria Adventista de Colombia, Medellín, Colombia.
} 
by changes in gross margin and profitability of their assets. On the other hand, an increase in selling prices adjusted for inflation and a substantial reduction in per capita bread consumption in the last 13 years, from 32 to 24 kilograms per inhabitant-year indicate a poor revenue performance and a poor use of its fixed assets. However, the behavior of the costs of raw materials and labor allows us to infer that employers were able to control production costs, despite the volatility of wheat's international prices.

Keywords: competitiveness, financial analysis, financial econometrics, bakery and pasta industry, profitability.

Clasificación JEL: G320

\section{Résumé}

L'industrie de la boulangerie et de pâtes en Colombie représente une des activités plus importante du secteur manufacturier, avec de ventes proches à 2,5 billions de pesos par an. Cependant, quelques facteurs critiques comme les prix des matières premières dans les marches internationaux, des aspects culturels, l'arrivée de fort concurrents transnationaux, et les adversités propres à l'industrie nationale, augmentent les risques pour les investisseurs. La rentabilité des capitaux propres montre une réduction progressive en passant de $14,1 \%$ en 2000 à $4,4 \%$ en 2013 ; de même, le modèle de régression linéal confirme ce comportement, qui s'explique en grande mesure par les changements de la marge brute et la rentabilité des actifs. D'autre part, une augmentation des prix de vente ajustés à l'inflation et une réduction substantielle de la consommation per capita de pain depuis 13 ans (de 32 à 24 kilos/habitant/an), indiquent une faible performance des revenus et de l'usage des actifs. Cependant, le comportement des coûts des matières premières et de la main d'œuvre permet de constater que les chefs d'entreprise ont contrôlé les coûts de productions, malgré la volatilité des prix internationaux du blé.

Mots Clés: compétitivité, analyse financière, économétrie financière, industrie de la boulangerie et des pâtes, rentabilité.

\section{Introducción}

Los gustos y preferencias del colombiano hacia otros bienes sustitutos del pan y la pasta, tales como: arepa, papa, plátano y yuca, constituyen un factor cultural de mucho arraigo que explica el bajo consumo. De otro lado, (Andi, 2015) y (Internacional Pasta Organization, 2014) los altos costos de la harina de trigo, por la baja producción nacional, hacen que Colombia sea un país dependiente de otros mercados, porque la demanda supera ampliamente la oferta, quedando a la deriva de los precios internacionales, con altos aranceles que encarecen la principal materia prima para el desarrollo de estas actividades. Banco de la república (2015), afirma que otras adversidades propias de toda la industria nacional como los altos costos logísticos por las deficiencias en la infraestructura vial, la obsolescencia tecnológica y en general la escasez de canales de distribución, obstaculizan el flujo eficiente de la materia prima y el producto terminado. Paralelamente, Cámara Fedemol (2015) sostiene los tratados de libre comercio han cambiado las condiciones del mercado para la industria colombiana; en particular la incursión de Bimbo en el sector panificador y de pastas desde la dé- cada de 1990 marcó un hito para una industria dominada tradicionalmente por productores nacionales como Ramo y Comapan, quienes eran considerados fuertes competidores con marcas muy posicionadas.

En la primera parte del documento se enmarca el componente teórico de la investigación, sustentado en las teorías actuales de competitividad, el análisis financiero y los modelos econométricos. Luego, se expone la metodología utilizada en la elaboración del modelo de regresión lineal múltiple por mínimos cuadrados ordinarios (MCO) y finalmente, el acápite de resultados permite desde el lenguaje financiero, identificar las principales causas que están afectando la rentabilidad de los inversionistas (ROE), con sus respectivas pruebas de significancia, estacionariedad, no multicolinealidad, causalidad y análisis gráfico, para dar la suficiente validez a los resultados obtenidos. El fin último, es hacer una contribución a la industria para mejorar los niveles de competitividad y atender problemáticas puntuales como la globalización de los mercados, la posición dominante de grandes industrias multinacionales y los déficits en la producción de materias primas fundamentales, entre otros. 


\section{Marco teórico}

\subsection{Competitividad}

Actualmente existe un mayor consenso sobre la teoría de la competitividad, en torno a los planteamientos de Porter (1991):

La competitividad está fundamentada en las bases microeconómicas de una nación: la sofisticación de las operaciones y estrategias de una compañía y la calidad del ambiente microeconómico de los negocios en la cual las compañías compiten. Entender los fundamentos microeconómicos de la competitividad es vital para la política económica nacional.

Lombana y Rosas (2009) plantean el nivel de análisis de la competitividad desde tres ámbitos: agregación, macro y micro. Para los propósitos de esta investigación el interés se centra en el nivel microeconómico que corresponde a los sectores, empresas y productos, cuya medición y análisis se sustenta cuantitativamente en la participación del mercado, indicadores de productividad y/o costo, márgenes de ganancia y/o beneficios netos. En efecto, para determinar la situación financiera de las empresas, se consideró un grupo de indicadores correspondientes al diagnóstico financiero que permite evaluar la liquidez, endeudamiento, rentabilidad y rotación del sector, tomando como referente para hacer comparaciones a toda la industria alimenticia en general.

\subsection{Análisis financiero}

Por diagnóstico financiero o análisis financiero puede entenderse el estudio que se hace de la información que proporciona la contabilidad y de toda la demás información disponible, para tratar de determinar la situación financiera de la empresa o de un sector específico de ésta. (García, 1999, p. 190). Para Van Horne y Wachowicz (2012), esta definición se origina en los estados financieros proporcionados por la disciplina contable y a partir de estos, se establecen diferentes tipos de relaciones entre sus cuentas, para obtener los indicadores financieros. La Figura 1 muestra los conceptos que agrupan los indicadores financieros de mayor uso en el diagnóstico financiero.
Figura 1. Indicadores o razones financieras

\begin{tabular}{l|r}
\hline $\begin{array}{l}\text { Liquidez: } \\
\text { Permite medir la } \\
\text { capacidad que tiene la } \\
\text { empresa para cancelar } \\
\text { sus obligaciones en el } \\
\text { corto plazo. }\end{array}$ & $\begin{array}{r}\text { Endeudamiento: } \\
\text { Miden la participación de } \\
\text { los acreedores en el } \\
\text { financiamiento de la } \\
\text { empresa. }\end{array}$ \\
$\qquad \begin{array}{c}\text { Clasificación de las } \\
\text { razones financieras }\end{array}$ \\
$\begin{array}{l}\text { Actividad o ratación: } \\
\begin{array}{l}\text { Mide la eficiencia en el } \\
\text { uso de los activos, según } \\
\text { la velocidad de la } \\
\text { recuperación. }\end{array} \\
\begin{array}{r}\text { Rendimiento o } \\
\text { rentabilidad: } \\
\text { que se administra la }\end{array} \\
\text { empresa, basado en la } \\
\text { capacidad de convertir las } \\
\text { ventas en utilidades para } \\
\text { sus dueños. }\end{array}$ \\
\hline
\end{tabular}

Fuente: Elaboración propia.

Para Cruz, Villarreal y Rosillo (2000) realizar el diagnóstico conlleva dos tipos de análisis: vertical y horizontal. El primero, relaciona cada una de las partes del estado financiero con respecto a una cifra base, para un mismo período y permite evaluar políticas, el tipo de empresa, la estructura financiera, márgenes de rentabilidad, etc. El segundo, permite analizar cambios en las cuentas individuales de los estados financieros, de un período a otro; es dinámico y las cifras se pueden expresar en forma de variaciones absolutas, porcentuales o razones.

Los indicadores financieros, se agrupan generalmente en cuatro clases, que permiten determinar la salud financiera de la empresa.

\subsection{Econometría}

Adicional al conocimiento de la teoría económica y financiera, es necesaria la práctica continua del desarrollo de los modelos econométricos estructurales y empíricos que permitan desarrollar la habilidad de seleccionar las variables adecuadas (Gómez, 2014). Según la metodología econométrica tradicional (Gujarati y Porter, 2009), el planteamiento de los modelos se rige por: planteamiento de la teoría, modelación matemática de la teoría, especificación del modelo econométrico, obtención de los datos, estimación de pa- 
rámetros, pruebas de hipótesis, pronóstico o predicción y uso del modelo.

El modelo básico de los MCO permite explicar el comportamiento de una variable dependiente a partir de varias variables explicativas o regresoras. La siguiente ecuación representa el modelo general:

$Y_{t}=\alpha+\sum_{i=1}^{k} \beta_{i} Y_{t-1}+\sum_{i=1}^{k} \gamma_{i} \chi_{t-1}+U_{t}$

Donde:

$Y_{t}$ : variable dependiente en $\mathrm{t}=0$

$Y_{t-1}:$ variables dependientes rezagadas $(t=-1$, $-2, \ldots)$

$\mathrm{X}_{\mathrm{t}-\mathrm{i}}$ : variables independientes actuales $(\mathrm{t}=0)$ y rezagadas

$\alpha, \beta, \gamma$ : coeficientes (intercepto y pendientes de las variables explicativas

$\mathrm{U}_{\mathrm{t}}$ : residuo de la regresión

La finalidad del modelo es replicar el comportamiento de los datos reales, lo que da lugar a la existencia de residuos o perturbaciones que muestran la diferencia entre los datos reales y el modelo, pero de cualquier forma se deben cumplir ciertos supuestos para hacer válidos los estimadores de la regresión:

1. El promedio aritmético de los residuos tiende a cero: $\mathrm{E}\left(\mathrm{U}_{\mathrm{i}}\right)=0$. Dado el valor de $\mathrm{X}_{\mathrm{i}^{\prime}}$ la media o el valor esperado del término de perturbación aleatoria $\mathrm{U}_{\mathrm{i}}$ es cero.

2. No multicolinealidad. Cov $\left(\mathrm{X}_{\mathrm{i}}, \mathrm{Y}_{\mathrm{j}}\right)=0$ para $i \neq j$. La covarianza entre las variables regresoras es cero. El análisis de las correlaciones entre las variables da una primera aproximación sobre qué tan cercanas o alejadas se encuentran las variables entre ellas; su magnitud entre 1 y -1 define su grado de dependencia, además el signo positivo (negativo) indican si la relación es directa (inversa). El valor cercano a cero indica independencia.

3. No correlación entre variables regresoras y residuos. $\operatorname{Cov}\left(\mathrm{X}_{\mathrm{i}}, \mathrm{Y}_{\mathrm{j}}\right)=0$. Las variables regresoras (Xs, Xs rezagadas, Ys rezagadas) y los residuos no deben estar correlacionados; su covarianza debe ser igual a cero.
4. No auto-correlación serial de los residuos $\operatorname{Cov}\left(U_{i}, U_{j}\right)=0$ para $i \neq j$. La covarianza entre los residuos debe ser igual a cero.

5. Homocedasticidad de los residuos. Var $\left(\mathrm{U}_{\mathrm{i}}=\sigma^{2}\right)$. La varianza de los residuos debe ser igual a un número constante positivo. Esto significa que la variación alrededor de la línea de regresión es la misma para todos los valores de X; es decir, no aumenta ni disminuye cuando varía $\mathrm{X}$.

6. Distribución normal de los residuos. $\mathrm{U}_{\mathrm{i}} \sim \mathrm{N}$ $\left(0, \sigma^{2}\right)$. Los residuos deben tener una distribución normal (media igual a cero y varianza)

\section{Metodología}

El tipo de investigación es aplicada y según sus características es correlacional causal, cuya población objeto de análisis corresponde a las empresas que reportaron información contable y financiera a la Superintendencia de Sociedades entre 2000 y 2013, según los datos del Sistema de Información y Reporte Empresarial (SIREM). En la Tabla 1 se especifican las variables consideradas en el modelo de regresión y sus respectivas fórmulas de cálculo. Posterior a la obtención de los estados financieros básicos (Balance General, Estado de Resultados y Estado de Flujos de Efectivo), se procedió a aplicar filtros entre 2000 y 2009 por códigos CIIU 1551 y 1552 que corresponden a "Elaboración de productos de panadería" y "Elaboración de macarrones, fideos, alcuzcuz y productos farináceos similares" respectivamente. Para los años 2010 a 2013 los filtros se aplicaron a los códigos 1081 y 1083, correspondientes a las mismas actividades, según la última reclasificación. Luego, se propone un modelo econométrico que determina las variables de mayor influencia en la ROE y para su validez se utilizó el software E-views en su versión 9.0, donde se desarrollaron las siguientes pruebas:

- Prueba de significancia general en términos de $\mathrm{R}^{2}$.

- Prueba de estacionariedad.

- Cumplimiento del supuesto de no multicolinealidad.

- Pruebas de causalidad.

- Prueba gráfica. 
Tabla 1. Definición de variables de la regresión Variable dependiente

\begin{tabular}{|c|c|}
\hline $\begin{array}{l}\text { Rentabilidad del } \\
\text { Patrimonio (ROE) }\end{array}$ & $\mathrm{ROE}=\frac{\text { Utilidad Neta }}{\text { Patrimonio }}$ \\
\hline \multicolumn{2}{|c|}{ Variable independiente } \\
\hline Margen Bruto (MB) & MB $=\frac{\text { Utilidad Bruta }}{\text { Ventas }}$ \\
\hline $\begin{array}{l}\text { Margen Operativo } \\
\text { (MO) }\end{array}$ & MO $=\frac{\text { Utilidad Operativa }}{\text { Ventas }}$ \\
\hline $\begin{array}{l}\text { Rentabilidad del } \\
\text { Activo (ROA) }\end{array}$ & ROA $=\frac{\text { Utilidad Operativa }}{\text { Activos Totales }}$ \\
\hline Liquidez (LI) & $\mathrm{LI}=$ Act. corriente - Pas.Corriente \\
\hline Prueba Ácida (PA) & $\mathrm{PA}=\frac{\text { Act. Corriente }- \text { Inventario }}{\text { Pas.Corriente }}$ \\
\hline Endeudamiento (EN) & $\mathrm{EN}=\frac{\text { Total Pasivo }}{\text { Total Activo }}$ \\
\hline
\end{tabular}

Fuente: Elaboración propia.

\section{Resultados y discusión}

\subsection{Diagnóstico financiero}

El análisis de los indicadores de rentabilidad muestra una disminución importante en sus principales indicadores, a pesar del comportamiento estable del margen bruto (entre $34 \%$ y $38 \%$ ). Al respecto se puede inferir que las variaciones en los costos de producción han sido consistentes con las variaciones en las ventas, a pesar de que en el 2013 se presentó el aumento más significativo de este indicador al reportar una utilidad bruta de $\$ 38,13$ por cada $\$ 100$ vendidos (Figura 2).

El rango del margen operativo se encuentra entre 3,3\% (año 2006) y 14,7\% (año 2001). Se destaca la reducción significativa de este indicador en los últimos tres años, lo que indica aumentos significativos en sus gastos de operación para ambos sectores.

De otro lado, uno de los resultados que más se destaca es la rentabilidad del activo, que refleja una reducción continua de su valor al pasar de 13,8\% en 2001 a 3,9\% en 2013, situación que contribuye significativamente a la destrucción de valor económico, porque al inicio del período de análisis $\$ 100$ de activos generaban $\$ 13,81$ de utilidades, mientras que en el último año la misma cantidad de activos produjo $\$ 3,99$.

Asimismo, la ROE evidencia un comportamiento más volátil que varía entre $0,5 \%$ en 2006 y $18,9 \%$ en 2001 , con una tendencia decreciente en los últimos cinco años. A manera de conclusión general, se puede afirmar sobre la rentabilidad de la actividad panificadora y de pastas que en los últimos 13 años ha presentado una disminución gradual de sus indicadores, sobre todo en la rentabilidad del activo (ROA) y la rentabilidad del patrimonio (ROE); esta situación se explica principalmente por un aumento en los gastos de operación

\section{Figura 2. Comportamiento de la rentabilidad entre 2000 y 2013}

Análisis de la rentabilidad

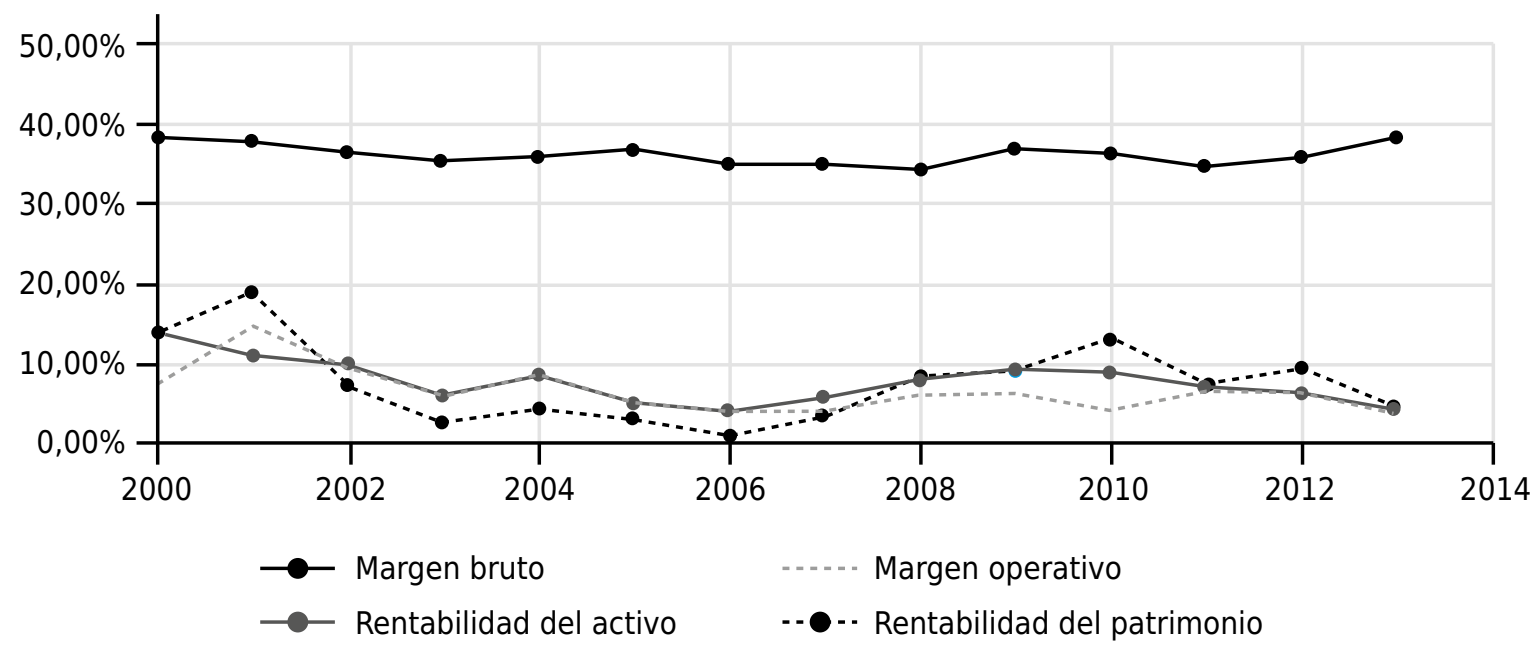

Fuente: elaboración propia con datos de SuperSociedades 
y un aumento en el nivel de endeudamiento que implican mayores gastos financieros y menos participación de los propietarios en la estructura financiera de sus negocios.

La Figura 3 muestra el comportamiento de la liquidez del sector de los últimos 13 años. Sus resultados reflejan un comportamiento estable entre 2000 y 2009, con un valor atípico de \$172.000 millones en el 2001. Los demás años indican que los activos corrientes exceden a los pasivos corrientes en niveles normales que varían entre $\$ 30.000$ y $\$ 96.000$ millones de pesos. Entre 2010 y 2013 los resultados muestran niveles excesivos de liquidez, sobre todo en el 2010, cuyo resultado indica que el activo corriente excede el pasivo del corto plazo en $\$ 366.000$ millones de pesos; no obstante este nivel, no afectó los indicadores de rentabilidad. Finalmente, este indicador regresa a niveles normales en el último año al ubicarse en $\$ 51.000$ millones.

Al evaluar la liquidez desde el indicador de prueba ácida, se infiere un manejo eficiente de sus inventarios, aunque la naturaleza del negocio presume altas rotaciones. En este sentido, el indicador muestra un comportamiento generalizado que oscila alrededor de uno; no obstante los resultados de los últimos tres años muestran una disminución importante, arrojando un valor de 0,70 para el úl- timo año, lo que quiere decir que la empresa dispone de $\$ 70$ de activo líquido, por cada $\$ 100$ de pasivos con vencimientos inferiores a un año.

Finalmente, el análisis del endeudamiento (Figura 4), muestra una tendencia alcista, sobre todo en los últimos diez años; mientras en el 2004 el sector registraba una financiación externa de $\$ 24$ por cada $\$ 100$ de activos, en el último año la proporción es de \$39. El año 2010 también evidencia una situación atípica en el nivel de endeudamiento al superar el $50 \%$.

\subsection{Modelo econométrico}

La Tabla 2 muestra la serie de datos de los indicadores financieros calculados entre 2000 y 2013, con base en la información suministrada por la Superintendencia de Sociedades. A partir de los resultados obtenidos en el diagnóstico financiero, se evaluó mediante el modelo de regresión lineal por MCO múltiple, cuáles son los que explican de la mejor manera el comportamiento de la rentabilidad de la inversión (ROE).

Para evaluar la validez del modelo de regresión se realizaron las siguientes pruebas:

Figura 3. Comportamiento de la liquidez entre 2000 y 2013

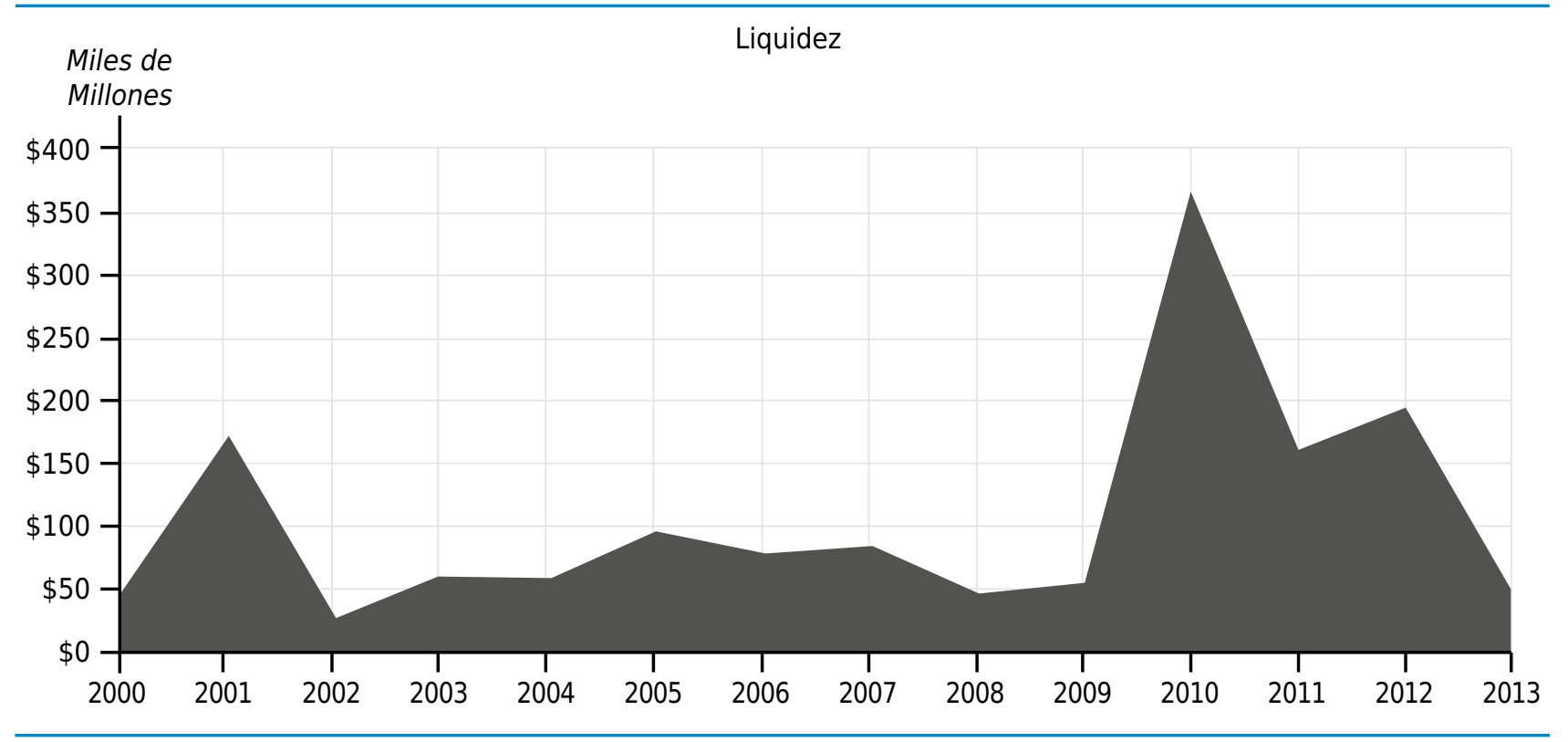

Fuente: Elaboración propia con datos de SuperSociedades 
Figura 4. Comportamiento del endeudamiento entre 2000 y 2013

Endeudamiento

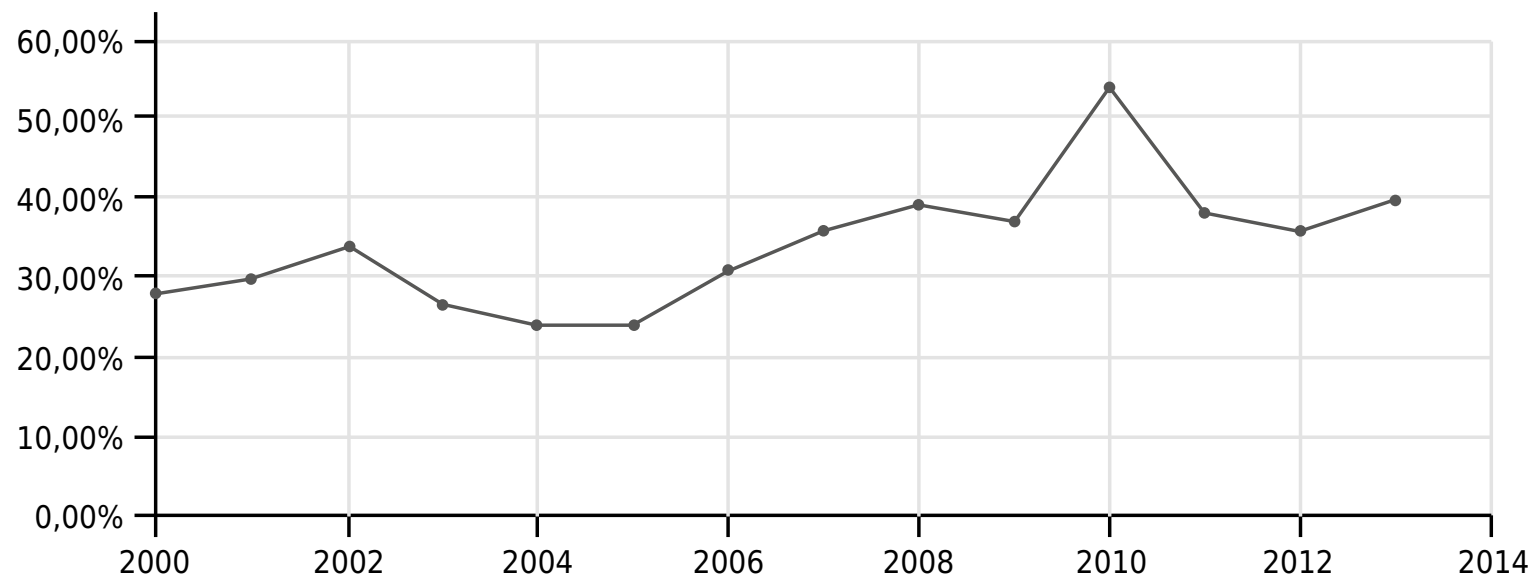

Fuente: Elaboración propia con datos de SuperSociedades

Tabla 2. Series de datos de los indicadores financieros

\begin{tabular}{c|c|c|c|c|c|c|c}
\hline Año & $\begin{array}{c}\text { Margen } \\
\text { bruto (\%) }\end{array}$ & $\begin{array}{c}\text { Margen } \\
\text { operativo } \\
\text { (\%) }\end{array}$ & $\begin{array}{c}\text { Rentabilidad } \\
\text { del activo } \\
\mathbf{( \% )}\end{array}$ & $\begin{array}{c}\text { Liquidez } \\
\text { (miles de } \$ \text { ) }\end{array}$ & $\begin{array}{c}\text { Prueba } \\
\text { ácida }\end{array}$ & $\begin{array}{c}\text { Endeuda- } \\
\text { miento } \\
\mathbf{\%})\end{array}$ & $\begin{array}{c}\text { Rentabili- } \\
\text { dad del } \\
\text { patrimonio } \\
\text { (\%) }\end{array}$ \\
\hline 2000 & 38,20 & 7,5 & 13,8 & $\$ 48.950 .796$ & 1,26 & 28,20 & 14,1 \\
2001 & 37,60 & 14,7 & 10,8 & $\$ 172.372 .267$ & 1,00 & 29,70 & 18,9 \\
2002 & 36,70 & 8,7 & 9,6 & $\$ 30.654 .101$ & 0,90 & 33,90 & 7,0 \\
2003 & 35,30 & 5,8 & 5,8 & $\$ 63.131 .066$ & 1,09 & 26,80 & 2,6 \\
2004 & 36,10 & 7,5 & 8,3 & $\$ 61.466 .055$ & 1,19 & 24,00 & 4,1 \\
2005 & 36,40 & 4,9 & 4,9 & $\$ 96.797 .669$ & 1,41 & 24,29 & 3,1 \\
2006 & 34,90 & 3,3 & 3,9 & $\$ 81.508 .796$ & 1,08 & 30,70 & 0,5 \\
2007 & 34,60 & 3,9 & 5,5 & $\$ 85.261 .126$ & 1,03 & 35,90 & 2,9 \\
2008 & 34,20 & 5,7 & 7,6 & $\$ 49.743 .271$ & 0,87 & 39,40 & 8,1 \\
2009 & 36,90 & 6,1 & 9,2 & $\$ 57.905 .759$ & 1,05 & 37,40 & 8,8 \\
2010 & 35,88 & 3,9 & 8,8 & $\$ 366.366 .034$ & 1,87 & 53,90 & 12,9 \\
2011 & 34,50 & 6,4 & 6,8 & $\$ 162.461 .189$ & 0,90 & 38,30 & 7,3 \\
2012 & 35,90 & 5,8 & 6,3 & $\$ 195.932 .255$ & 0,93 & 35,90 & 9,0 \\
2013 & 38,10 & 4,1 & 3,9 & $\$ 51.007 .590$ & 0,73 & 39,90 & 4,4 \\
\hline
\end{tabular}

Fuente: Elaboración propia con datos de SuperSociedades

1. Estructura de las series originales (prueba de significancia)

Para evaluar la estructura de la serie, se utilizó la prueba de significancia general de una regresión múltiple en términos de $R^{2}$, utilizando la siguiente regla de decisión (Gujarati y Porter, 2009, p. 242):

Dado el modelo de regresión con K variables:

$Y_{i}=\beta_{i}+\beta_{2} X_{2 i}+\beta_{3} X_{3 i}+\cdots+\beta_{x} X_{k i}+U_{i}$

(Ec. 2)
Para probar la hipótesis:

$H_{0}=\beta_{2}=\beta_{3}=\ldots \beta_{\mathrm{k}}=0$

Frente a:

H1: no todos los coeficientes de pendiente son simultáneamente cero.

Calcular:

$$
F=\frac{\frac{R^{2}}{K-1}}{\frac{1-R^{2}}{n-k}}
$$


Si $F>F_{\alpha(k-1, n-k)}$ se rechaza la hipótesis nula (Ho), o si el valor $\mathrm{p}$ del F obtenido es lo bastante bajo, rechace Ho. Los cálculos iniciales de la prueba permiten determinar el valor de $\mathrm{R}^{2}$, utilizando análisis de varianza (ANOVA) y sus resultados se muestran en la Tabla 3

\begin{tabular}{l|l}
\hline \multicolumn{2}{c}{ Tabla 3. Resultados del análisis de varianza } \\
\hline \multicolumn{1}{c}{ Estadísticas de la regresión } & \multicolumn{1}{c}{ Valor } \\
\hline Coeficiente de correlación múltiple & 0,965530017 \\
Coeficiente de determinación $\mathrm{R}^{\wedge} 2$ & 0,932248214 \\
$\mathrm{R}^{\wedge} 2$ ajustado & 0,874175255 \\
Error típico & 0,018201684 \\
Observaciones & 14 \\
\hline \multicolumn{2}{c}{ Fuente: Elaboración propia }
\end{tabular}

El coeficiente de determinación indica que $93,2 \%$ de las variaciones en la rentabilidad del patrimonio (ROE) son explicadas por las variables regresoras consideradas en el modelo, para un total de 14 observaciones.

La Tabla 4 muestra los resultados del análisis de varianza, necesarios para la aplicación de la prueba F.

Los grados de libertad de la Tabla 4 permiten obtener el numerador (k-1) y denominador (n-k) para aplicar dicha prueba. Estos valores corresponden a los grados de libertad correspondientes a la regresión (6) y a los residuos (7). En efecto, la aplicación de la fórmula anterior, permite obtener un valor para F de 16,0530, cuyo resultado lo explica la siguiente igualdad:

$$
F=\frac{\frac{0,932248}{6}}{\frac{1-, 932248}{7}}=16,0530
$$

Para un nivel de significancia del 5\%, el valor de $\mathrm{F}$ con los grados de libertad mencionados antes, se obtiene como resultado 3,87 , según los resultados de las tablas de esta distribución. Como 16,0530>3,87 indica que se rechaza la hipótesis nula (Ho) y se asume como verdadero la hipótesis alterna (Ha), en efecto no todos los coeficientes dependientes son simultáneamente cero y se concluye que el intercepto y la tendencia son estadísticamente significativos.

\section{Pruebas de estacionariedad}

Para determinar la estacionariedad de cada variable se aplicó la prueba de Elliot-Rothenberg-Stock ${ }^{1}$ (ERS), que examinan la hipótesis nula (Ho) y la hipótesis alterna con un coeficiente de significancia del $5 \%$, quedando así el planteamiento de esta prueba:

Ho: la rentabilidad del patrimonio (ROE) tiene raíz unitaria

Ha: la rentabilidad del activo (ROE) es estacionaria

Lo anterior indica que si el resultado de la prueba es menor al valor crítico al $1 \%$, $5 \%$ y $10 \%$, se rechaza la hipótesis nula.

La Tabla 5 muestra los resultados correspondientes a la prueba de estacionariedad para la rentabilidad del patrimonio (ROE).

La aplicación de la prueba ERS da como resultado un valor del estadístico de 17,20205 que no es menor a los valores críticos al 1\%, 5\% y $10 \%$, en efecto se acepta la hipótesis nula y se concluye que la serie no es estacionaria. Este mismo procedimiento se aplicó para todas las demás series y la Tabla 6 resume los resultados, de la cual se infiere que ninguna de ellas es estacionaria.

\begin{tabular}{|c|c|c|c|c|c|}
\hline & $\begin{array}{l}\text { Grados de } \\
\text { libertad }\end{array}$ & $\begin{array}{l}\text { Suma de } \\
\text { cuadrados }\end{array}$ & $\begin{array}{c}\text { Promedio de los } \\
\text { cuadrados }\end{array}$ & $\mathbf{F}$ & Valor crítico de F \\
\hline Regresión & 6 & 0,031910381 & 0,005318397 & 16,05305166 & 0,000899118 \\
\hline Residuos & 7 & 0,002319109 & 0,000331301 & & \\
\hline Total & 13 & 0,03422949 & & & \\
\hline
\end{tabular}

1 Su aplicación se hace con las siguientes consideraciones: (1) intercepto significativo, (2) intercepto y tendencia significativa, (3) ninguno es significativo. 
Tabla 5. Prueba de Elliot-Rothenberg-Stock

Null Hypothesis: D(RENTABILIDAD_DEL_PATRIMONIO) has a unit root

Exogenous: Constan, Linear Trend

Lag lenght: 0 (Spectral OLS AR based on SIC, maxlag=2)

Sample (adjusted): 20012013

Included observations: 13 after adjustments

\begin{tabular}{|c|c|c|}
\hline & P-statistic \\
\hline Elliot-Rothenberg-S & est Statistic & 17.20295 \\
\hline \multirow[t]{3}{*}{ Test critical values } & $1 \%$ level & 4.220000 \\
\hline & $5 \%$ level & 5.720000 \\
\hline & $10 \%$ level & 6.770000 \\
\hline
\end{tabular}

*Elliot-Rothenberg-Stock (1996, Table 1)

Warning: Test Critical values calculated for 50 observations and may not be accurate for a sample size of 13

\begin{tabular}{l|l}
\hline HAC corrected variance (Spectral OLS & 0.001802
\end{tabular} autoregression)

Fuente: Elaboración propia con datos de E-views 9.0

Tabla 6. Consolidación de resultados de la prueba de Elliot-Rothenberg-Stock

Nivel de significancia (1\%, 5\%, 10\%)

Valores críticos $(4,2200 ; 5,7200 ; 6,7700)$

\begin{tabular}{l|c|c|c}
\hline \multicolumn{1}{c|}{ VARIABLE } & P-Estad & $\begin{array}{c}\text { Acepta } \\
\text { Ho }\end{array}$ & $\begin{array}{c}\text { ¿Es } \\
\text { estacionaria? }\end{array}$ \\
\hline Rentabilidad del activo & 13,96269 & $\mathrm{Si}$ & No \\
Margen bruto & 24,02213 & $\mathrm{Si}$ & No \\
Margen operativo & 170,1606 & $\mathrm{Si}$ & No \\
Rentabilidad del patrimonio & 17,20295 & $\mathrm{Si}$ & No \\
Liquidez & 19,04289 & $\mathrm{Si}$ & No \\
Prueba ácida & 15,78876 & $\mathrm{Si}$ & No \\
Endeudamiento & 14,58432 & $\mathrm{Si}$ & No \\
\hline
\end{tabular}

Fuente: Elaboración propia con datos de E-views 9.0

Al concluir que todas las series tienen raíces unitarias, como es de esperarse de la mayoría de series económicas y financieras, se procedió a expresar las variables en logaritmos, con el fin de evitar errores en los estimadores de la regresión. La Tabla 7 muestra la serie de datos transformada en sus logaritmos.

\section{Evaluación del supuesto de no multicolinealidad}

Para evaluar la dependencia que pueda existir entre las variables regresoras y cumplir con el supuesto de no multicolinealidad, se procedió a observar el coeficiente de correlación entre dichas variables. "Si este valor es superior a 0,8, la multicolinealidad es un problema grave... y son comparativamen- te bajas, si son inferiores a 0,5 , no obstante las correlaciones altas son suficientes pero no necesarias para la existencia de multicolinealidad" (Gujarati y Porter, 2009, p. 338). La Tabla 8 muestra la matriz de los coeficientes de correlación entre los diferentes pares de variables.

$\mathrm{Al}$ evaluar los coeficientes de correlación entre las distintas variables regresoras, se encuentra que en su mayoría tienen valores inferiores a 0,5 excepto la relación margen operativo (MO)-rentabilidad del activo (ROA), cuyo valor es 0,7144 , lo cual es explicable si se considera que este último depende en gran medida de los resultados de la operación del negocio (ventas, costos de producción y gastos de operación). En el mismo sentido, la rentabilidad del patrimonio (ROE) muestra una relativa alta correlación con la rentabilidad del activo (ROA), situación explicada por la estrecha relación que existe entre estos dos indicadores. A pesar de estos últimos resultados se decidió no descartar ninguna variable, porque sus valores son tolerables al no superar el valor de 0,8 .

\section{Prueba de causalidad de Granger}

Para determinar en qué medida $x_{i}$ contiene información relevante para predecir $y_{j}$, se aplicó la prueba de Granger, según el siguiente valor de la distribución F:

$$
F=\frac{\left(S C R_{R}-\right.}{\left.S C R_{N}\right)}
$$

donde

$\mathrm{SCR}_{\mathrm{R}}$ : Suma de cuadrados de residuales de la regresión restringida.

$\mathrm{SCR}_{\mathrm{NR}}$ : Suma de cuadrados de residuales de la regresión no restringida.

m: Número de términos rezagados.

k: Número de parámetros estimados en la regresión no restringida.

Para probar la hipótesis:

$H_{0}: \alpha_{\mathrm{i}}=0, i=1,2, \ldots, n$

Frente a:

$H_{\alpha}: \alpha_{\mathrm{i}} \neq 0, i=1,2, \ldots, n$ 
Tabla 7. Series de variables transformadas en logaritmos

\begin{tabular}{|c|c|c|c|c|c|c|c|}
\hline Año & $\begin{array}{l}\text { Rentabilidad } \\
\text { del activo }\end{array}$ & Margen bruto & Margen bruto & $\begin{array}{c}\text { Margen } \\
\text { operativo }\end{array}$ & $\begin{array}{c}\text { Rentabilidad } \\
\text { del patrimonio }\end{array}$ & Liquidez & $\begin{array}{c}\text { Endeuda- } \\
\text { miento }\end{array}$ \\
\hline 2000 & $-1,98$ & $-0,96$ & $-0,96$ & $-2,59$ & $-1,96$ & 17,71 & $-1,27$ \\
\hline 2001 & $-2,23$ & $-0,98$ & $-0,98$ & $-1,92$ & $-1,67$ & 18,97 & $-1,21$ \\
\hline 2002 & $-2,34$ & $-1,00$ & $-1,00$ & $-2,44$ & $-2,65$ & 17,24 & $-1,08$ \\
\hline 2003 & $-2,85$ & $-1,04$ & $-1,04$ & $-2,85$ & $-3,65$ & 17,96 & $-1,32$ \\
\hline 2004 & $-2,48$ & $-1,02$ & $-1,02$ & $-2,59$ & $-3,20$ & 17,93 & $-1,43$ \\
\hline 2005 & $-3,01$ & $-1,01$ & $-1,01$ & $-3,01$ & $-3,48$ & 18,39 & $-1,42$ \\
\hline 2006 & $-3,23$ & $-1,05$ & $-1,05$ & $-3,40$ & $-5,31$ & 18,22 & $-1,18$ \\
\hline 2007 & $-2,91$ & $-1,06$ & $-1,06$ & $-3,23$ & $-3,51$ & 18,26 & $-1,02$ \\
\hline 2008 & $-2,57$ & $-1,07$ & $-1,07$ & $-2,86$ & $-2,52$ & 17,72 & $-0,93$ \\
\hline 2009 & $-2,39$ & $-1,00$ & $-1,00$ & $-2,80$ & $-2,43$ & 17,87 & $-0,98$ \\
\hline 2010 & $-2,43$ & $-1,02$ & $-1,02$ & $-3,25$ & $-2,04$ & 19,72 & $-0,62$ \\
\hline 2011 & $-2,68$ & $-1,06$ & $-1,06$ & $-2,74$ & $-2,62$ & 18,91 & $-0,96$ \\
\hline 2012 & $-2,76$ & $-1,03$ & $-1,03$ & $-2,84$ & $-2,40$ & 19,09 & $-1,02$ \\
\hline 2013 & $-3,22$ & $-0,96$ & $-0,96$ & $-3,20$ & $-3,13$ & 17,75 & $-0,92$ \\
\hline
\end{tabular}

Fuente: Elaboración propia con datos de E-views 9.0

Tabla 8. Matriz de correlaciones

\begin{tabular}{c|c|c|c|c|c|c|c}
\hline & ROA & MB & MO & ROE & LIQ & PA & END \\
\hline ROA & 1,00000 & 0,36325 & 0,71446 & 0,78675 & $-0,0123$ & 0,25936 & 0,00863 \\
MB & 0,36325 & 1,00000 & 0,39650 & 0,41422 & $-0,1897$ & 0,01694 & $-0,17735$ \\
MO & 0,71446 & 0,39650 & 1,00000 & 0,61415 & $-0,0940$ & $-0,1697$ & $-0,36062$ \\
ROE & 0,78675 & 0,41422 & 0,61415 & 1,00000 & 0,2486 & 0,06621 & 0,32492 \\
LIQ & $-0,01237$ & $-0,18977$ & $-0,09403$ & 0,24867 & 1,00000 & 0,46051 & 0,38720 \\
PA & 0,25936 & 0,01694 & $-0,16975$ & 0,06621 & 0,46051 & 1,00000 & $-0,03647$ \\
END & 0,00863 & $-0,17735$ & $-0,36062$ & 0,32492 & 0,38720 & $-0,0364$ & 1,00000 \\
\hline
\end{tabular}

Fuente: Elaboración propia con datos de E-views 9.0

Si el valor de $\mathrm{F}$ calculado excede al valor $\mathrm{F}$ crítico para el nivel de significancia seleccionado, se rechaza la hipótesis nula (Ho). La Tabla 9 muestra los resultados de la prueba que permiten determinar si las cinco variables regresoras tienen causalidad sobre la rentabilidad del patrimonio (ROE), utilizando dos rezagos.

El primer resultado de la Tabla 9 prueba la hipótesis de que el endeudamiento causa la rentabilidad del patrimonio; se observa que el valor probable del estadístico $\mathrm{F}$ es 0 , 1097, que resulta menor al valor observado $(3,08168)$; esta situación indica que se debe rechazar la hipótesis nula (Ho), de allí se afirma que los coeficientes son diferentes de cero y en consecuencia, la variable endeudamiento aporta información para explicar la rentabilidad del patrimonio (ROE). El segundo resultado muestran un valor probable 0,9601 superior al estadístico $\mathrm{F}$ $(0,04097)$, en consecuencia el coeficiente es igual a cero, por tanto se acepta la hipótesis nula (Ho) y se podría afirmar que la liquidez (LI) no causa rentabilidad en el patrimonio (ROE), es decir, la liquidez no aporta información relevante para explicar el comportamiento del ROE.

Al evaluar los demás resultados, se puede concluir: endeudamiento (EN), margen bruto (MB), margen operativo (MO) y rentabilidad del activo (ROA), tienen causalidad sobre la rentabilidad del patrimonio y aportan información importante para explicar su comportamiento; mientras que la Liquidez (LI) y la prueba ácida (PA) no implican causalidad sobre la variable explicada, lo que confirma la teoría financiera al afirmar que la ROE depende en gran medida de la eficiencia que se tenga en la operación 
Tabla 9. Prueba de causalidad de Granger

\begin{tabular}{l} 
Pairwise Granger Causality Tests \\
Date: 05/06/15 Time: 09:43 \\
Sample 2000 2013 \\
Lags: 2 \\
\hline
\end{tabular}

Fuente: Elaboración propia con datos de E-views 9.0

del negocio, respecto a la participación de ellos; es decir, que depende de la capacidad que tengan sus inversiones para generar las utilidades que finalmente son trasladadas al patrimonio.

\section{Resultados de la regresión}

La construcción del modelo de regresión lineal, una vez validadas las pruebas anteriores, arrojó como resultado un coeficiente de determinación $\mathrm{R}^{2}$, con un valor de 0,8555 e indica que $85,5 \%$ de los cambios ocurridos en la rentabilidad del patrimonio (ROE), son explicados por los cambios en las variables regresoras. La Tabla 10 muestra los resultados de la regresión a través del software.

Asimismo, del reporte anterior se deriva la siguiente ecuación que muestra los resultados obtenidos en la regresión:

$$
\begin{aligned}
& \mathrm{ROA}=3,0472+5,7164 * \mathrm{MB}+0,4963 * \mathrm{ROA} \\
& -0,6849 * \mathrm{PA}+1,3457 * \mathrm{EN} \quad \text { (Ec. 8) } \\
& \text { donde } \\
& \text { ROA = Rentabilidad del activo } \\
& \mathrm{MB}=\text { Margen bruto }
\end{aligned}
$$

$$
\begin{aligned}
& \text { MO = Margen operativo } \\
& \text { ROE = Rentabilidad del patrimonio } \\
& \text { PA = Prueba ácida } \\
& \text { EN = Endeudamiento }
\end{aligned}
$$

Estos indican que todas las variables regresoras, con excepción de la PA tienen un efecto positivo sobre la rentabilidad del patrimonio (ROE), siendo la de mayor influencia el margen bruto (MB), cuyo valor explica que un aumento de $1 \%$ en el MB produce un aumento de 5,7\% en el ROE. Seguidamente, la ROA también tiene un efecto importante sobre la variable explicada (un aumento del $1 \%$ en la ROA, produce un aumento de 1,5\% en la ROE); en su orden le siguen el endeudamiento (EN) y el margen operativo (MO) y con un efecto adverso la PA indica que un aumento del $1 \%$ en este indicador ocasiona una disminución del 0,63\% en la ROE. De otro lado, el estadístico F (F-Statistic $=6,9192)$ es superior a su valor probable Prob (F-statistic $=0,01117)$, de allí se infiere también que conjuntamente las variables regresoras son estadísticamente significativas. Finalmente, la Figura 5 también permite concluir que el modelo proyectado se ajusta bien a los datos históricos. 
Tabla 10. Resultados de la regresión lineal por el MCO

Dependent Variable: RENTABILIDAD_DEL_PATRIMONIO

Method: Least Squares

Date: 05/06/15 Time: 09:31

Sample: 20002013

Included observations: 14

\begin{tabular}{l|c|c|c|c}
\hline Variable & Coefficient & Std. Error & t-Statistic & Prob. \\
\hline C & 3.047200 & 7.939731 & 0.383791 & 0.7125 \\
RENTABILIDAD_DEL_ACTIVO & 1.503442 & 1.036409 & 1.450626 & 0.1902 \\
PRUEBA_ACIDA & -0.684913 & 1.283776 & -0.533515 & 0.6102 \\
MARGEN_OPERATIVO & 0.496324 & 1.076403 & 0.461095 & 0.6587 \\
MARGEN_BRUTO & 5.716457 & 4.133720 & 1.382884 & 0.2092 \\
LIQUIDEZ & 0.371879 & 0.359823 & 1.033507 & 0.3357 \\
ENDEDUDAMIENTO & 1.345795 & 1.217714 & 1.105181 & 0.3056 \\
\hline R-squared & 0.855585 & Mean dependent var & $-2,898562$ \\
Adjusted R-squared & 0.731801 & S.D. dependent var & 0.925365 \\
S.E. of regression & 0.479227 & Akaike info criterion & 1.673568 \\
sum squared resid & 1.607609 & Schwarz criterion & 1.993096 \\
log likelihood & -4.714974 & Hannan-Quinn criter & 1.643990 \\
F-statistic & 6.911925 & durbin-Watson sta & 2.700730 \\
Prob(F-statistic) & 0.011170 & & \\
\hline
\end{tabular}

Fuente: Elaboración propia con datos de E-views 9.0

Figura 5. Datos proyectados y reales 2000-2013

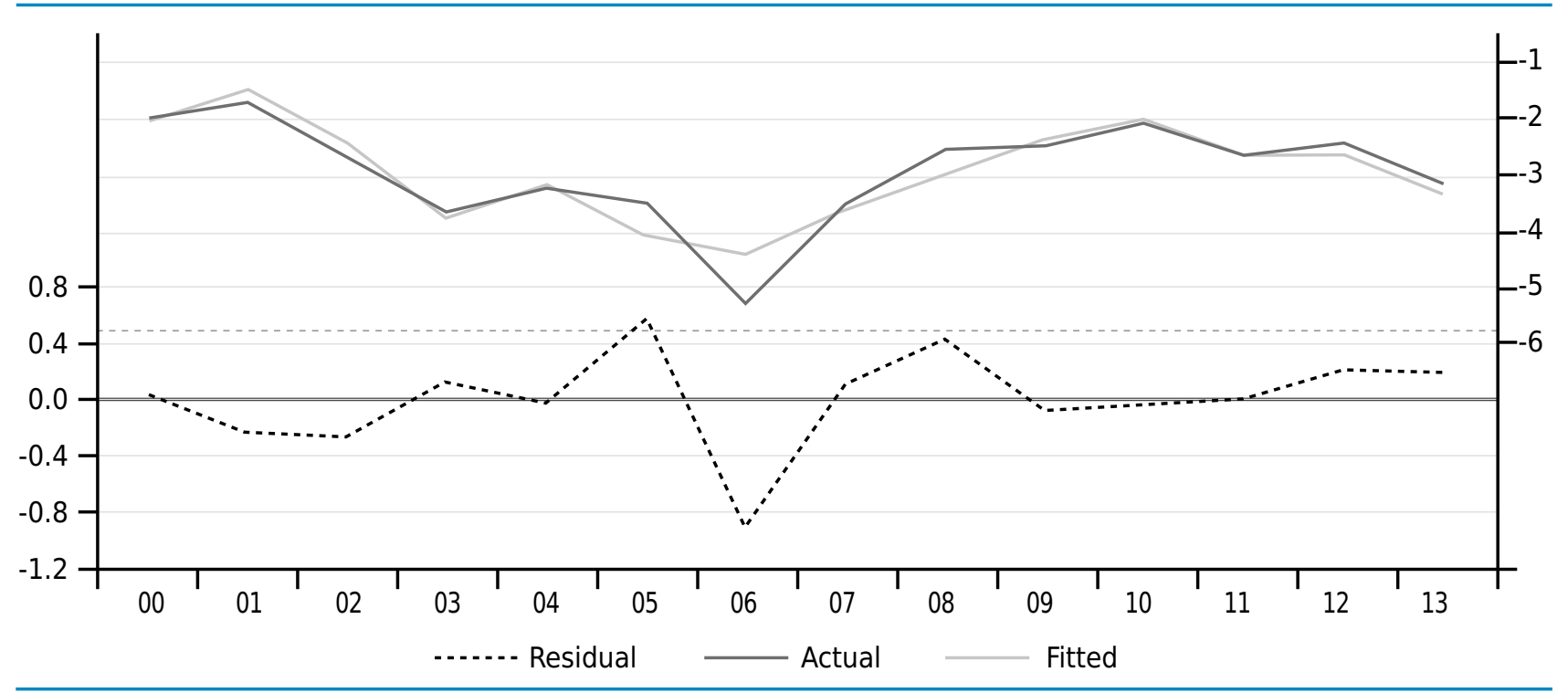

Fuente: Elaboración propia con datos de E-views 9.0

\section{Conclusiones}

Es imperativo para la industria panificadora y de pastas colombiana apropiarse de los conocimientos y habilidades de naturaleza gerencial, financiera, productiva, tecnológica y de mercado, que permita establecer una relación costo-beneficio-efectividad en cada uno de sus procesos. Esta condición es ne- cesaria para alcanzar el posicionamiento de sus productos y atender las nuevas amenazas y oportunidades del mercado nacional e internacional (David, 2013).

Específicamente, desde la disciplina financiera, el desarrollo de la investigación permite inferir que la ROE evidencia un comportamiento volátil, cuyo valor máximo y mínimo 
fueron $0,5 \%$ en 2006 y $18,9 \%$ en 2001 , con una tendencia decreciente en los últimos cinco años. Asimismo, los resultados del modelo econométrico explica que el detrimento de la ROE está marcado por la falta de control en los gastos de operación y la disminución en la capacidad de los activos para generar utilidades. Estos resultados se validan con los obtenidos en el análisis de los índices de productividad sectorial y diagnóstico financiero, de acuerdo con las siguientes explicaciones:

En términos de ventas se concluye que los años de mayor actividad en el período de análisis fueron 2004 y 2008, mientras que en 2005 se obtuvieron los resultados más bajos para ambas industrias, con un crecimiento promedio de $17 \%$ anual, explicado principalmente por los ajustes en los precios, porque otros estudios demuestran una reducción del consumo per cápita de pan en los 13 últimos años al quedar en 25 kilos-habitante-año.

El análisis de los costos de materias primas y el consumo intermedio ratifican al año 2008 como el período de mayor crecimiento y el 2005 una baja producción y baja demanda En este sentido los costos de las materias primas han tenido una variación consistente con el volumen de ventas.

El análisis de la mano de obra también indica una variación proporcional con las ventas, a pesar de un aumento en términos de salario real, con un promedio superior a otras actividades económicas, además de una estabilidad en la tercerización de las operaciones. De allí también se concluye que la actividad panificadora y de pastas constituye una fuente importante de empleo en la economía colombiana, según (Dane, 2008) la ocupación llegó a 26.652 personas en 2010 y una persona dedicada a estas actividades obtuvo una remuneración promedio de $\$ 804.000$ mensuales en el año 2000 (incluyendo prestaciones sociales) y \$1'401.000 en 2011.

El comportamiento de los costos de materias primas y mano de obra permiten inferir que los empresarios tuvieron control sobre los costos de la producción y sus resultados explican la estabilidad del margen bruto, cuyos valores se movieron entre 34\% y 38\%; mientras que el margen operativo ha sido más volátil, lo que deriva en menos control sobre sus gastos operacionales de administración y ventas, sobre todo entre 2010 y 2013.
La ROA ha tenido un deterioro sostenido en los últimos años, lo que indica menor eficiencia en el uso de sus activos: mientras en el 2000, \$100 de activos generaban $\$ 13,81$ de utilidades operativas, en el 2013 sólo produjo $\$ 3,99$ de utilidades.

El nivel de endeudamiento muestra que en los últimos diez años los propietarios han disminuido su participación en los activos de la empresa, porque el sector se financia cada vez más con recursos externos y menos con recursos propios. No obstante, el aumento del apalancamiento no ha favorecido a los inversionistas en sus utilidades.

El sector muestra indicadores adecuados de liquidez a lo largo del período de análisis, excepto en 2010 con un excedente de 366.000 millones de pesos y la prueba ácida indica una alta rotación de sus inventarios.

\section{Referencias}

Asociación Nacional de Industriales. (2015). Situación actual de las perspectivas de la harina de trigo en Colombia. Recuperado de http://www. andi.com.co/SitEco/Documents/Balance\%20 2014\%20y\%20 perspectivas\%202015.pdf

Banco de la República. (2015). Evolución de la Balanza de pagos y Posición de Inversión Internacional. Recuperado de http://banrep.gov.co/sites/default/files/paginas/ibp_ene_mar_2015.pdf

Cámara Fedemol. (2011). Informe de la XXVIII Feria Alimentaria. Recuperado de www.andi.com. co/downloadfile.aspx?Id=DFE29751-F932-401D

Consejo Económico para América Latina y el Caribe. (2014). Balance preliminar de las economías de América Latina y el Caribe. Recuperado de http://repositorio.cepal.org/bitstream/handle/11362/37344/Colombia_es.pdf?sequence $=7$

Cruz, S., Villarreal, J., y Rosillo, J. (2000). Finanzas Corporativas. Bogotá, Colombia: Thomson.

DANE. (2008). Muestra mensual manufacturera. Recuperado de http://www.dane.gov.co/files/investigaciones/boletines $/ \mathrm{mmm} / \mathrm{bol}$ _espec_alim bebida07.pdf

David, F. (2013). Conceptos de Administración Estratégica. Ciudad de México, México: Pearson.

García, O. (1999). Administración Financiera. Medellín, Colombia: Prensa Moderna. 
Gómez, A. (2014). Econometría aplicada a finanzas y mercado de capitales. Bogotá, Colombia: Universidad Libre de Colombia.

Gujarati, D., y Porter, D. (2009). Econometría. New York, USA: Mc Graw Hill.

Internacional Pasta Organization. (2012). The World Pasta Industry Status Report 2012. Recuperado de http://www.internationalpasta.org/ resources/World\%20Pasta\%20Industry\%20 Survey/IPOstatreport2013.pdf
Lombana, J., y Rosas, S. (2009). Marco analítico de la competitividad. Pensamiento y Gestión, (7), 28-31.

Porter, M. (1991). La ventaja competitiva de las naciones. Buenos Aires, Argentina: Vergara.

Superintendencia de Sociedades. (2015, Marzo). Sistema de Información y Registro Empresarial. Recuperado de www.supersociedades.gov.co.

Van Horne, J., y Wachowicz, J. (2012). Fundamentos de Administración Financiera. Stanford, Ciudad de México, México: Mc Graw Hill.

¿Cómo citar este artículo? - How to quote this article?

Delgado-Vélez, L.D. (2016, junio). Análisis econométrico de la rentabilidad de los inversionistas en la industria panificadora y de pastas colombiana, 2000-2013. Cuadernos de Administración, 32(55), 19-32. 